Musées, Patrimoine et Culture scientifiques et techniques

$121 \mid 2009$

janvier - février 2009

\title{
La rénovation du musée de l'École nationale vétérinaire de Maisons-Alfort
}

Christophe Degueurce

\section{OpenEdition \\ Journals}

Édition électronique

URL : http://journals.openedition.org/ocim/261

DOI : 10.4000/ocim.261

ISSN : 2108-646X

Éditeur

OCIM

Édition imprimée

Date de publication : 1 janvier 2009

Pagination : 5-11

ISSN : 0994-1908

Référence électronique

Christophe Degueurce, "La rénovation du musée de l'École nationale vétérinaire de Maisons-Alfort », La Lettre de I'OCIM [En ligne], 121 | 2009, mis en ligne le 01 janvier 2011, consulté le 01 mai 2019. URL http://journals.openedition.org/ocim/261 ; DOI : 10.4000/ocim.261 


\section{Du cobinet d'Alfort au musée de l'École nationale vétérinaire de Maisons-Alfort}

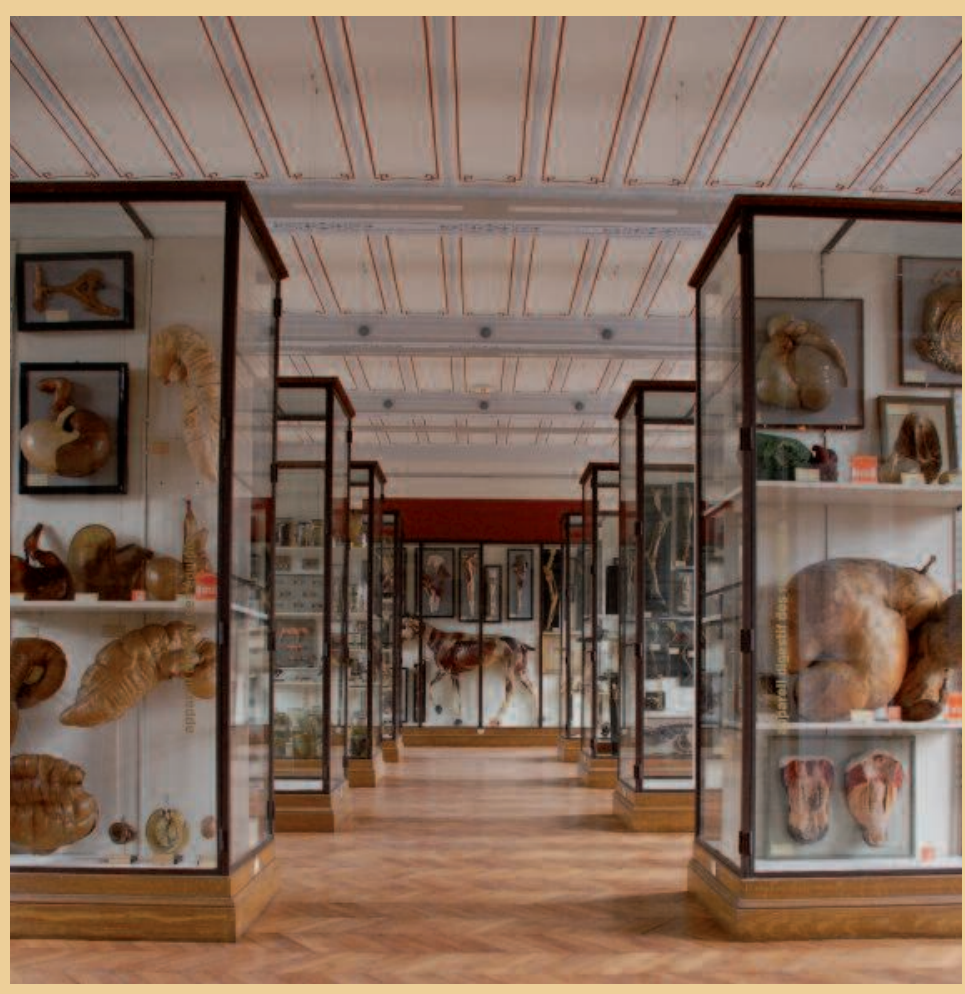

Le musée au début du $X X^{e}$ siècle : la salle d'Anatomie

Le musée de l'École nationale vétérinaire de MaisonsAlfort est l'héritier du cabinet du roi que Claude Bourgelat, fondateur des deux premières écoles vétérinaires au monde, constitua à l'École d'Alfort en 1766. C'était alors un cabinet d'Histoire naturelle et d'Anatomie comparée alimenté par les travaux pratiques des étudiants. Il fut, dès sa création, accessible au public. La qualité des pièces qui s'y accumulèrent rapidement lui valut une notoriété dans toute l'Europe et nous disposons de descriptions très précises qui en furent faites à cette époque. Les débuts du cabinet furent marqués par le nom d'Honoré Fragonard, premier directeur et premier professeur d'Anatomie de l'école, auquel on doit des écorchés toujours présentés aujourd'hui. Il s'agit de cadavres humains et animaux qui furent disséqués puis momifiés pour permettre l'enseignement de l'Anatomie. Il ne s'agit pas à proprement parler de pièces à vocation artistique mais de spécimens scientifiques, dédiés à la pédagogie et bénéficiant d'une approche esthétique s'inscrivant dans la continuité de l'histoire de l'Anatomie.

Le cabinet devait subir les vicissitudes de la Révolution ; l'école royale vétérinaire d'Alfort fut menacée de nombreuses fois de délocalisation et de suppression, et ses collections diminuèrent pendant la Révolution. Il s'emplit à nouveau de préparations dès cette période troublée passée. Il fut déplacé vers un nouveau bâtiment en 1829. Sa surface s'en trouva accrue et il accueillit de nouvelles collections notamment de Pathologie qui décidèrent l'administration à lui donner le nom de « cabinet des collections ». Il ne semble pas que le public y ait été accueilli ; le cabinet fonctionnait plutôt comme une sorte de réserve organisée à des fins d'enseignement. Les professeurs venaient y prélever des pièces qui servaient ensuite à la démonstration en amphithéâtre. Le très fort développement de l'École d'Alfort au XIX ${ }^{\mathrm{e}}$ siècle, sa participation aux grands mouvements scientifiques comme la Physiologie expérimentale ou encore les développements de l'Infectiologie, précédants et contemporains de l'épopée pasteurienne, participèrent au renforcement de son aura et se traduisirent pas une augmentation significative de ses collections. Le cabinet des collections devint, à partir des années 1860, trop étroit pour accueillir l'ensemble des collections. Il fut donc décidé en 1882 de le déménager une nouvelle fois.

Il devait prendre place dans un nouveau bâtiment en 1900 et prit le nom de «musée ». Ce nouveau musée était à la fois un outil pédagogique et un moyen de communication. Il servait aux élèves qui venaient $y$ apprendre l'Anatomie et la Pathologie. Mais c'était également un lieu de prestige destiné à montrer aux étrangers la puissance de l'école qui avait accompagné Louis Pasteur dans bien des découvertes touchant aux maladies animales. Dès les années 1920, les étudiants se firent de plus en plus rares au musée et son ouverture fut réservée aux visiteurs de marque. Le musée resta fermé au public jusqu'en 1991, date à laquelle il rouvrit ses portes sous le nom de «musée Fragonard », s'appuyant sur les étudiants de l'école pour assurer son fonctionnement.

Les collections avaient été remaniées sommairement pour permettre un abord de la collection par le grand public. Le nom même du musée focalisait l'attention sur l'anatomiste et les 21 pièces sensées être de sa main, plaçant au second plan le reste des 4200 objets accessibles à la visite. Ce positionnement permit un développement constant de la fréquentation si bien que le musée devint un acteur incontournable de la culture scientifique et du tourisme en Val-de-Marne. 


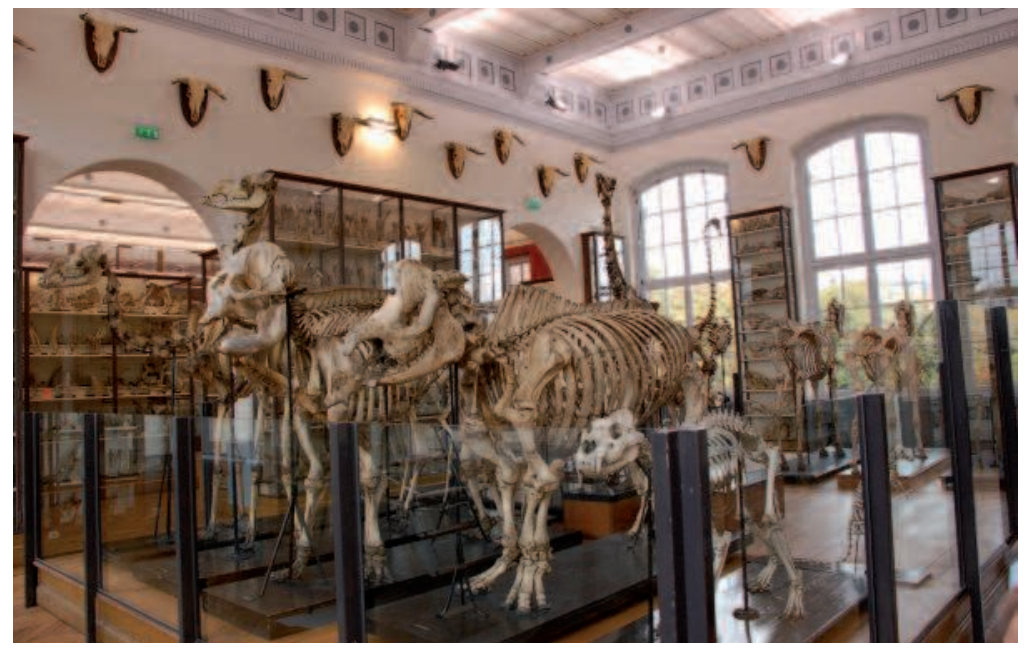

La salle des squelettes

(๑) Christophe Degueurce

leurs systèmes vasculaires. Cette dégradation détermina les principales actions du musée pendant les années qui suivirent à savoir, la recherche de partenaires financiers pour gérer un projet de réhabilitation du musée, l'École vétérinaire d'Alfort n'étant pas en mesure, seule, de financer une opération d'une telle importance, et la recherche de préconisations en matière de conservation préventive des écorchés.

\section{La recherche de partenaires financiers}

Fort de sa visibilité dans le domaine du tourisme, le musée Fragonard a candidaté à l'appel d'offres lancé par le Pôle touristique régional des Boucles de la Marne. Cette structure résulte du partenariat de la Région Île-de-France avec la délégation régionale au Tourisme (Préfecture de Région) et le Conseil général du Val-de-Marne. Son objectif est de développer l'activité touristique dans l'est parisien en s'appuyant sur le territoire des Boucles de la Marne, riche d'un patrimoine naturel original en Île-de-France et bénéficiant d'une image associée aux loisirs et à la détente (guinguettes, promenades en barques, piscines naturelles...). C'est donc tout naturellement que la proximité de l'École d'Alfort avec le confluent de la Marne et de la Seine a été utilisé pour proposer un projet de site touristique dédié à l'animal domestique. Ce projet a été retenu et a bénéficié d'une étude de faisabilité qui a recommandé la mise en œuvre d'un projet en trois phases : une phase initiale correspondant au sauvetage des collections du musée actuel et à la mise en valeur touristique du musée, une seconde phase dédiée à la construction d'un nouveau bâtiment permettant d'étendre l'exposition permanente et de développer des zones d'exposition temporaire, et une troisième phase s'appuyant sur la rénovation d'un amphithéâtre historique pour développer une politique de conférences à caractère scientifique ${ }^{(1)}$.

\section{La recherche de préconisations en matière de conservation préventive des écorchés}

Rénover le musée pour permettre leur conservation impliquait de déterminer les conditions dans lesquelles conserver ces spécimens. Mais Honoré Fragonard avait pris garde de ne jamais révéler une technique dont on apprend, en lisant certaines pièces de sa correspondance, qu'elle était originale, permettait la réalisation de très belles injections vasculaires et protégeait les écorchés de la dégradation par les insectes consommateurs de ce type de pièces anatomiques. Comment espérer pouvoir sauvegarder des œuvres quand on ne connaît pas leur nature intime et donc les conditions à mettre en œuvre pour les stabiliser ? Nous avons donc mis en place une démarche scientifique visant à comprendre la façon dont les écorchés de Fragonard avaient été réalisés.

Lopportunité est venue d'une autorisation de tournage sollicitée par National Geographic Channel, dans le cadre de la série télévisée The mummies road show. Le principe de cette émission était celui d'un reportage sur le travail de deux scientifiques découvrant peu à peu les secrets des momies. Un accord a alors été passé pour mener une investigation de L'Homme à la mandibule, en échange de la réalisation de tests sur l'injection et le vernis recouvrant cette pièce. Ce choix s'est fondé sur la grande qualité technique de cette pièce marquée par des injections vasculaires spectaculaires. L'étude a été conduite sur place et a enchâ̂né les examens physique, radiographique et endoscopique. Des échantillons de produits d'injection ont été prélevés afin de mener lidentification des pigments et du vernis et envoyés à deux laboratoires nord américains. Les résultats contradictoires qui sont ressortis de l'analyse des mêmes échantillons ont conduit à solliciter ensuite le Laboratoire de Recherche des Monuments Historiques (Paulette Hugon), puis le 


\section{Les particularités de la technique de Fragonard}

Les Écorchés de Fragonard font partie des très rares pièces de ce type à avoir traversé les siècles. On sait par une lettre écrite par Fragonard en 1792 qu'il avait mis au point une technique originale d'injection du système vasculaire qui permettait la réalisation de magnifiques pièces, et qu'il disposait d'un moyen prévenant leur destruction par les insectes ${ }^{(1)}$. Les recherches effectuées entre 2003 et 2005 ont révélé un certain nombre de secrets de l'anatomiste. Tout d'abord, Fragonard, comme tous les anatomistes, portait la plus grande attention au choix des corps. Un des critères semble être l'état de maigreur qui facilitait l'injection et la dissection. Ensuite il injectait le système artériel via le cœur, après réalisation d'une thoracotomie. Cette voie lui assurait la mise en pression nécessaire au succès de l'opération.

Plus surprenant fut la découverte de la composition de la masse d'injection. On attendait de la cire ; on trouva surtout du suif de mouton mélangé à de la résine de pin et à une huile essentielle. Labsence de cire explique probablement la grande facilité d'utilisation de cette technique. Le suif de mouton fond à basse température, à la différence de la cire d'abeille qui fond aux environs de $70^{\circ} \mathrm{C}$. Il était donc aisé à Fragonard de faire fondre du suif de mouton dans un récipient, d'y ajouter de la résine de pin, et une huile essentielle pour obtenir un mélange fluide, facilement injectable dans un corps tiède. Cette technique devint la règle au XIXe siècle.

Les systèmes artériel et veineux étaient marqués avec des couleurs différentes, respectivement du rouge et du bleu. On a également pu observer que Fragonard, s'il injectait un mélange teinté avec du vermillon dans les artères, ne chargeait pas linjection destinée aux veines avec un pigment bleu qui en aurait augmenté la densité. Cette différence de charge pigmentaire entre artères et veines s'explique aisément ; les veines étant pourvues de valvule, la résistance à l'injection est plus importante que dans les artères.
L'anatomiste n'a donc pas pris le risque de rendre son mélange plus visqueux en le chargeant de pigments. La différence de teinte était suffisante pour quil puisse aisément distinguer pendant la dissection les artères des veines.

Une fois le corps injecté, Fragonard procédait à la dissection selon une méthode classique. Les organes difficiles à conserver, comme les poumons, les intestins, l'encéphale, étaient ôtés. Venait ensuite la phase de préservation ; le corps était probablement plongé dans l'alcool pour être déshydraté. L'étape suivante était la mise en position et le séchage. Fragonard utilisa probablement un cadre, où l'ensemble des moyens d'accrochage disponible dans la pièce pour positionner sa tête et ses bras. Les muscles et les vaisseaux étaient maintenus en place par des aiguilles qui sont toujours visibles. Il faut donc imaginer l'anatomiste travaillant les oreilles et les lèvres de l'homme pour leur donner l'attitude agressive qui impressionne tant aujourd'hui. Le même type de procédé a dû être adopté pour le cavalier et sa monture.

Venait ensuite la phase ultime de la préparation. Les artères comme les veines étaient d'abord peintes pour les souligner et, une fois le corps prêt, il était verni avec un produit contenant de la térébenthine de Venise, résine du mélèze, qui présente l'intérêt majeur de jaunir et non de noircir. Et il est probable que l'utilisation de ce dernier produit ne soit pas étrangère à la préservation des écorchés de l'attaque des insectes « destructeurs de toute collection » comme disait Fragonard dans sa lettre de 1792.

Au bilan, cette étude nous a permis de saisir certaines spécificités de la méthode de Fragonard, particularités qui expliquent les commentaires élogieux de ses contemporains sur ses réalisations et surtout leur longévité hors normes ${ }^{(2)}$.

(1) Fragonard, Delseuze, Landrieux. Lettre à l'Assemblée nationale, juillet 1792. Archives nationales F17 1318, dos sier 4 .

(2) Degueurce, C. Les écorchés de Fragonard : une recette de longévité, La Recherche, n414, 2007, pp. 66-73.
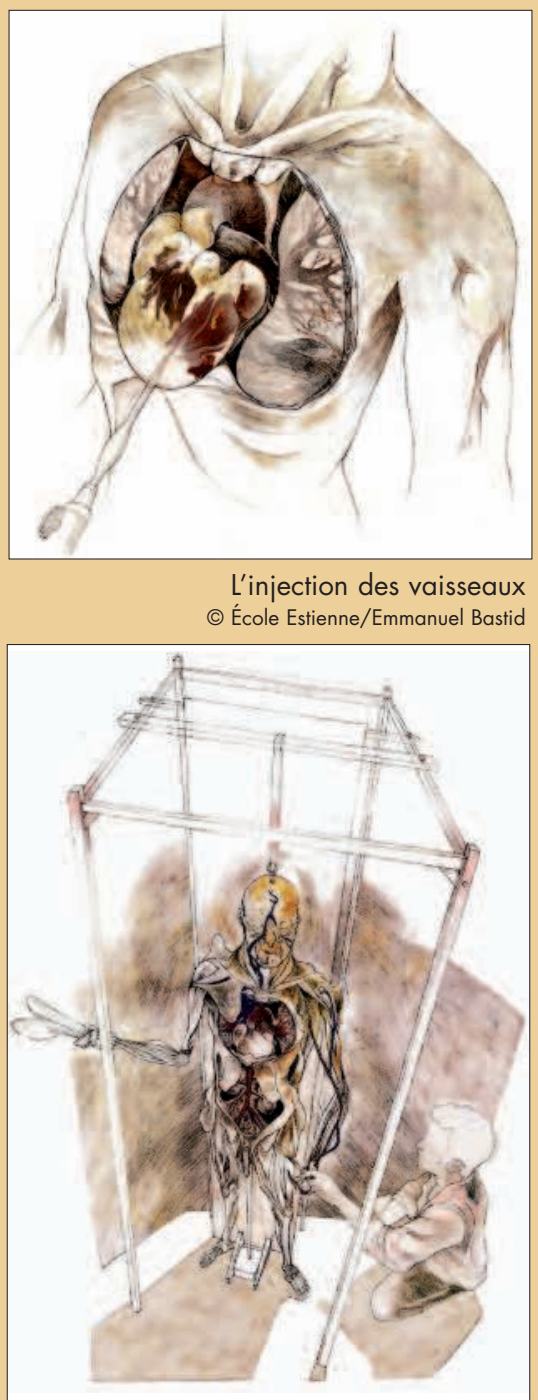

L'application du vernis à base de térébenthine de Venise (c) École Estienne/Emmanuel Bastid

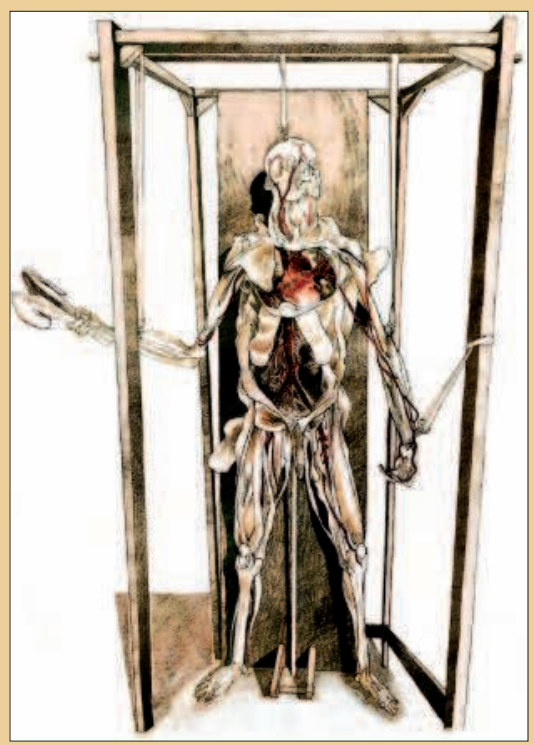

Le positionnement du corps dans un cadre (c) École Estienne/Emmanuel Bastid 
LETIAM de l'Institut universitaire de Technologie de Paris 11-Orsay (Sung Vo Dhui, Jean Bleton, Alain Tchapla), ceci dans le but de les confirmer et de les compléter. Sung Vo Dhui a d'ailleurs réalisé une partie de sa thèse de $3^{\text {e }}$ cycle en Chimie analytique sur l'étude des masses d'injection des écorchés (2).

Ces études ont révélé une bonne partie des secrets de Fragonard et nous ont permis d'édicter des recommandations en matière de conservation préventive de ses Écorchés. Le fait que l'injection soit essentiellement faite de suif de mouton nous a conduit à modifier l'atmosphère dans laquelle ils demeurent en cherchant à maintenir l'ambiance locale à $18^{\circ} \mathrm{C}$ (3) et au maximum à $50 \%$ d'hygrométrie ${ }^{(4)}$.

Ces deux éléments concomitants, la démarche de projet avec le Pôle touristique des Boucles de la Marne et la démarche scientifique dans une perspective de conservation préventive, ont conduit à l'élaboration du projet de rénovation, première phase du projet de pôle culturel et scientifique dédié à l'animal domestique. Les partenaires institutionnels ont assuré le financement de l'objectif initial du projet ; il s'agissait d'une intervention minimale visant à sauver les collections et à assurer la mise en tourisme du site. Pas de fioritures, pas de restitution des décors, d'amélioration de l'éclairage ; l'essentiel reposait sur la climatisation et la signalétique du musée. Cette mise de fonds initiale a été fondamentale : elle a permis de rendre crédible auprès de mécènes la perspective de la rénovation complète du musée. La notoriété des Écorchés de Fragonard et l'intervention des pouvoirs publics ont apporté une forte légitimité à la campagne de collecte de mécénat qui fut faite au cours de l'année scolaire 2006-2007 auprès d'entreprises de notre secteur d'activité (laboratoires pharmaceutiques vétérinaires), d'entreprises implantées localement, de fondations intervenant dans le domaine du patrimoine... Le résultat fut positif, changea les perspectives du projet et conduisit les collectivités territoriales à abonder cette nouvelle somme. Dès lors, la rénovation complète du musée était envisageable. Elle a commencé en décembre 2007 et s'est terminée en octobre 2008.

\section{Le choix de ramener le musée à un état proche de celui de 1902}

Bien évidemment, nous nous sommes posés la question d'une restructuration complète du musée Fragonard qui le projetterait dans un aspect plus contemporain. Mais la collection actuelle est fondée sur un principe accumulatif; chaque vitrine comprend un grand nombre d'objets se rapportant à un thème, avec très peu de commentaires disponibles. Le musée renferme ainsi, sur $500 \mathrm{~m}^{2}$, plus de 4200 pièces. La tentation aurait pu être de dé-densifier le musée et de garder moins de pièces qui auraient été mieux commentées. Finalement, notre choix a été de mener une restitution, de replacer le musée dans l'état qui était le sien lors de son inauguration, en 1902. Ce choix s'est fondé d'abord sur une étude réalisée auprès du public en 2004 qui nous a montré que la plupart des visiteurs, notamment les étrangers, venaient ici pour s'immerger dans le cadre suranné d'une collection du XIX e siècle. De nombreux visiteurs viennent là non pas pour les pièces qu'ils y verront, à part les Écorchés bien évidemment, mais pour admirer une architecture intérieure marquée par le rythme des vitrines, les dimensions des salles, l'organisation des objets et l'esthétique qui en résulte... Le musée est un lieu de patrimoine en lui-même, avant même les messages pédagogiques qui pourraient y être diffusés. À une heure où bien des musées anciens se sont transformés, il nous a semblé que ce lieu pouvait avoir vocation à être un témoignage d'une époque aspirant à l'exhaustivité. En somme, un « musée de musée » dont la facture patrimoniale assurera, nous l'espérons, la conservation et le développement dans le futur. Il s'agit donc d'un pari sur l'avenir.

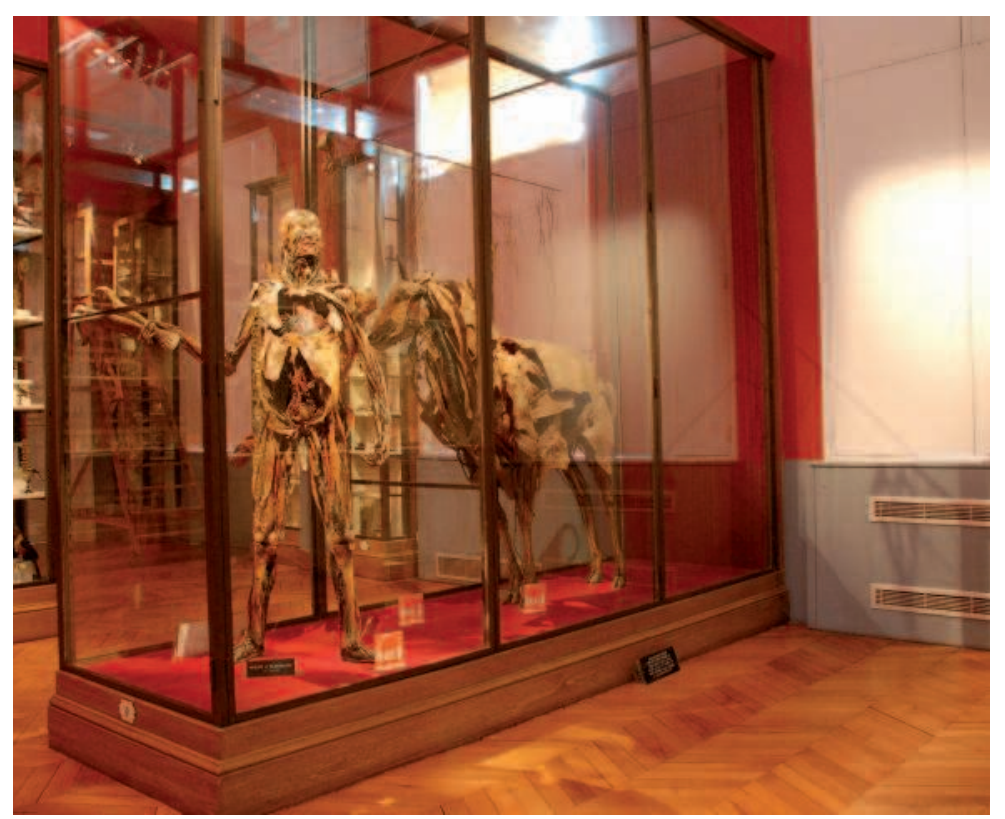

La vitrine L'Homme à la mandibule : écorché humain réalisé par Fragonard en station érigée et tenant dans sa main droite une mandibule de cheval (à droite le système de climatisation). (c) Christophe Degueurce 
S'ajoutait à ces considérations la difficulté de remanier en profondeur un musée dans un espace contraint. Pas de réserves à ce stade du projet donc pas de lieu où placer les objets retirés du musée principal. Et que dire du coût de l'investissement associé à un remaniement complet (déplacement des collections, création de vitrines...) et du coût de fonctionnement induit par la nécessité du renouvellement du discours ? Nous n'en avions pas, en première intention, les moyens et mieux valait sanctuariser le musée actuel, sauver les collections en maintenant l'ouverture au musée tout en cherchant à développer les phases ultérieures du projet global qui apporteraient cette dimension d'actualité.

Par ailleurs, je reste convaincu qu'une collection de type accumulatif permet une grande richesse du discours. La collection peut être le support de visites portant sur des thèmes très variés sans nécessiter de remaniement et c'est une multitude de discours qui peuvent être ainsi conçus en toute liberté. Une grande chance, notamment dans le partenariat avec les établissements d'enseignement. Même sans guide, chacun peut y trouver son compte et le visiteur peut venir plusieurs fois en redécouvrant à chaque visite de nouvelles pièces. Ceci ne veut pas dire que la collection est immuable. Elle a été entièrement repensée pour coller au message retenu pour la visite guidée ; ceci s'est fait avec les étudiants de l'École d'Alfort et a nécessité trois années de réflexion et de travail (2002 à 2004) avec une remise en état des vitrines, peinture des fonds et un réagencement des collections. Cependant, le résultat n'est pas visible, insoupçonnable pour la personne abordant le musée pour la première fois car cette restructuration a répondu aux principes de l'organisation initiale du musée. Il ne s'agissait donc que de déplacement d'ensembles de pièces, de modification des thématiques des vitrines, sans aucune dé-densification.

\section{Le programme de rénovation}

Il comprenait des modifications structurelles indispensables au plan de conservation préventive à mettre en œuvre pour sauvegarder les Écorchés. C'est ainsi que toutes les anciennes fenêtres ont été remplacées par des neuves dotées de verres filtrant les rayons ultra-violets. Une cloison isolante a été créée, qui sépare le cabinet de curiosités du reste du musée. La salle des Écorchés a ainsi pu être climatisée, limitant les dépenses énergétiques aux seuls espaces indispensables. Cette cloison crée une séparation physique qui permet au visiteur de percevoir nettement le

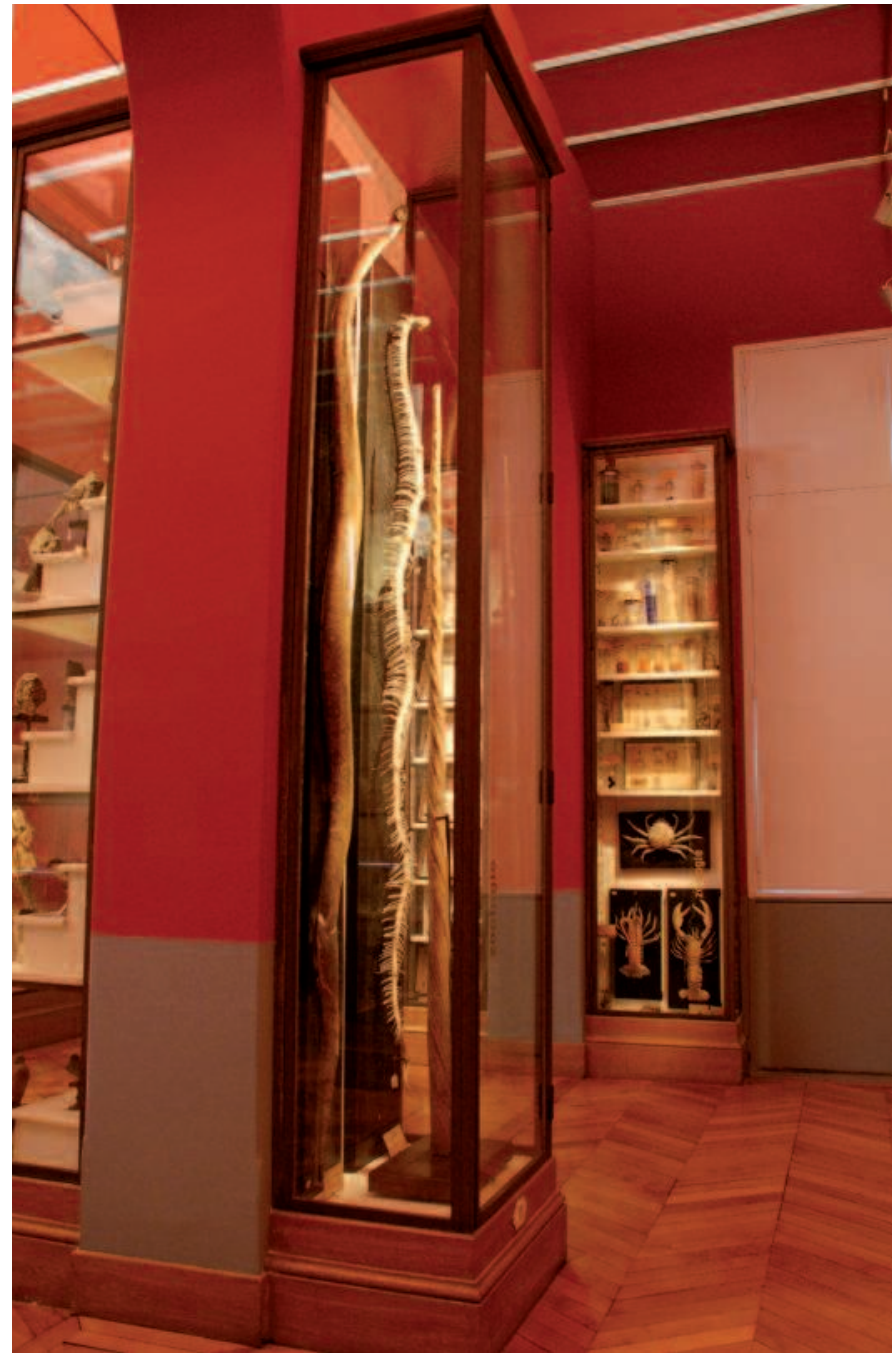

Détail d'une vitrine de Zoologie du cabinet de curiosités, après rénovation (c) Christophe Degueurce

changement d'ambiance. Les nécessités de conservation des Écorchés ont ainsi servi la muséographie. Tout au fond du musée, le visiteur arrive face à la grande paroi de verre granité qui lui cache la vue des Écorchés. Il doit pousser les portes de verre pour pénétrer dans le cabinet du roi et c'est là qu'il peut découvrir les quelque deux cents pièces qui ont traversé les siècles. Le passage dans cette quatrième salle est une rupture nette du point de vue de l'ambiance : la climatisation en modifie l'atmosphère, les murs sont peints en rouge sombre, des rangées de spots mettent en lumière des écorchés, les murs présentent des dessins expliquant comment ces spécimens anatomiques furent réalisés.

L'autre partie du programme concernait plus le traitement architectural global du reste du musée et l'accueil du public. C'est ainsi que des couleurs sombres, de l'ocre rouge et du gris foncé, ont été 
replacées sur les murs. Les décors qui ornaient les plafonds en 1900 ont été remis en place sur les poutres et sur les voûtins. L'éclairage a été entièrement refait pour mettre en valeur les collections comme les décors intérieurs du musée.

Une attention particulière a été portée à l'accueil du public. Une banque d'accueil a été installée à l'entrée du musée ; une signalétique est en fin de conception. Une visite audioguidée français/anglais a été créée qui permet au visiteur de s'approprier une centaine de pièces.

Pour sceller ce retour dans le passé, le musée a changé de nom et repris celui qui était le sien lors de son transfert en 1900 en redevenant "musée de l'École vétérinaire de Maisons-Alfort ». Le terme de « musée Fragonard » était restrictif car il focalisait l'attention sur seulement 11 des 4200 pièces présentées au public. Reste que le nom de Fragonard est ancré dans les consciences et qu'il faudra travailler la communication pour éviter les confusions. Le musée a rouvert ses portes le $1^{\text {er }}$ novembre 2008. Les étudiants ont été formés. Les plannings sont en place. Souhaitons lui le succès qu'il mérite et que ces travaux permettent de concilier la conservation d'une collection scientifique vulnérable et la venue des visiteurs.

\section{Notes}

(1) Cette étude a été réalisée par le cabinet In Situ (Sylvie-Marie Scipion).

(2) Vo Dhuy, S. Mise au point de méthodes analytiques pour la caractérisation de la matière organique constituante d'objets du patrimoine culturel. Thèse de $3^{\text {e }}$ cycle, université Paris 11, 2007.

(3) Le choix de maintenir la température à $18^{\circ} \mathrm{C}$ a été pris en considérant qu'une grande partie du suif est ramollie dès $20^{\circ} \mathrm{C}$. Nous avons donc choisi de nous placer dans une limite basse, compatible avec le confort des visiteurs, un des éléments fondamentaux étant la suppression des variations brutales qui avaient conduit aux fontes.

(4) L’hygrométrie doit être contrôlée car les écorchés sont des pièces conservées par déshydratation. 